\title{
Three-Dimensional Transport Theory: Evaluation of Analytical Expressions of Williams and verification of MCNP
}

Jeho Jeong ${ }^{+}$Nathan E. White ${ }^{(a)}$, Sudarshan K. Loyalka ${ }^{(a)} *$

(a) Nuclear Science and Engineering Institute

University of Missouri

Columbia, MO 65211

+ Present Affiliation

The Sloan Kettering Institute

New York, N.Y.

*Corresponding Author

Sudarshan K. Loyalka

LoyalkaS@missouri.edu

Phone: +1 (573) 882-3568

Fax: $\quad+1(573) 884-4801$

Nuclear Science and Engineering Institute

E2433 Lafferre Hall

University of Missouri

Columbia, MO 65211 


\begin{abstract}
"Three-dimensional transport theory: an analytical solution of an internal beam searchlight problem, I", Annals of Nuclear Energy, 36(8), 1256-1261 (2009) by Williams extends the range of analytical solutions, and the associated development of techniques, numerical results and analysis near singularities. The final integrals are not easy to evaluate as the integrands are highly oscillatory, singular and also on infinite range. We report here some further numerical evaluations of expressions of Williams, and also compare these with those of Williams and Ganapol and Kornreich. The numerical results compare very well. The disagreements are very rare, and even then in the fifth decimal place. We are also able to explore the nature of the results near singularities in conformity with the results of Williams. We also verify MCNP-5, the widely used Monte Carlo code against these analytical results. We have found that MCNP is easily able to provide results within $0.1 \%$ deviation from the "exact" results for most cases, and within $1 \%$ for almost all cases. It is challenged near the singularities, however, where the deviations are larger.
\end{abstract}




\section{Introduction}

Williams [2009] has stated,

"One of the most challenging remaining problems in analytical solutions of the transport equation must be that concerning multi-dimensional geometry. When the transport equation involves more than one space variable there is an increase in complexity due to interaction of the effects caused by these additional variables. The earliest exact solution known to the author on three- dimensional transport theory is due to Elliott (1952, 1955). This work describes the problem of a point source on the surface of a half-space; i.e. an early form of the so-called searchlight problem. Later work involved a variety of source configurations and even the quarter space problem, although admittedly this did not lead to a closed form solution (Lam and Leonard, 1971). Multidimensional solutions are notoriously difficult to obtain and the usual procedure is to take Fourier transforms in the two, infinite transverse directions, and a Laplace transform in the semi-infinite direction. This leads to a solution either by the Wiener-Hopf technique or as the solution of a singular integral equation.... Whilst the Wiener-Hopf method generally enables one of the inversions to be done analytically, the algebraic complexity of the Fourier transform usually prevents any neat inversion. The quest for such solutions is well worth while however since they form the basis of essential benchmark problems for validating large transport theory computer codes. The techniques and philosophy of such techniques and associated numerical methods has been discussed by Ganapol (2008a)."

He elaborates further,

"In pursuit of the above goals, Ganapol and Kornreich (2008) have recently developed a technique based on the FN method for solving one-speed transport problems involving interior mono-directional beam sources. Such sources cover point, multi-ring and disk geometries with the particles directed towards the surface. In the present work we wish to extend the scope of the Ganapol-Kornreich work to three-dimensional problems and also to introduce some useful ideas for reducing the numerical problems which arise due to the presence of Bessel functions in the inverse Hankel transform. Some numerical results are given but the emphasis is on technique." 
While Prof. Williams was finishing his subject paper, one of us (Loyalka) had occasion to work with him and carry out some independent numerical evaluations of the integrals involved by using techniques different than he was using. Prof. Ganapol had also made his computer program available for verification. The details of our evaluations, as well as some additional work we carried out later towards verification of MCNP-5 for the subject problems were not published. We are taking this opportunity now to provide those results together with some recent refinements. We note that for the ring source our results agree fully with those of Williams, while for the point source our results agree fully with those of Ganapol and Kornreich.. 


\section{Analytic Results and their Numerical Evaluation}

The problems considered by Williams and Ganapol and Kornreich are defined by the equations:

$$
\begin{aligned}
& \mu \frac{\partial I(x, y, z, \mu, \varphi)}{\partial z}+\sqrt{1-\mu^{2}}\left(\cos \varphi \frac{\partial}{\partial x}+\operatorname{sins} \varphi \frac{\partial}{\partial y}\right) I(x, y, z, \mu, \varphi) \\
& +I(x, y, z, \mu, \varphi)=\frac{c}{4 \pi} I_{0}(x, y, z)+S(x, y) \delta\left(z-z_{0}\right) \delta\left(\mu-\mu_{0}\right) \delta\left(\varphi-\varphi_{0}\right)
\end{aligned}
$$

Where, $I(x, y, z, \mu, \varphi)$ is the angular flux of particles, the scattering is isotropic and distances have been scaled in terms of mean free path. Also,

$$
I_{0}(x, y, z)=\int_{0}^{2 \pi} d \varphi \int_{-1}^{1} d \mu I(x, y, z, \mu, \varphi)
$$

is the scalar flux (we will use the nomenclature "density" also in the following). The boundary condition is:

$$
I(x, y, 0, \mu, \varphi)=0, \quad \mu>0, \varphi \in(0,2 \pi)
$$

Williams has expressed the solution of our interest here as:

$$
I_{0}\left(\rho, 0 \mid z_{0}\right)=\frac{1}{2 \pi} \int_{0}^{\infty} d k k J_{0}(k \rho) \bar{S}(k) \bar{G}_{0}\left(k \mid z_{0}\right)
$$

Where, the Green's function $\bar{G}_{0}\left(k \mid z_{0}\right)$ is given in Williams in terms the $\mathrm{H}$ function and $\bar{S}(k)$ is the Fourier Transform of the source (Table 1), and for ring and disk sources involves Bessel functions of $0^{\text {th }}$ or $1^{\text {st }}$ order: 
Table 1: Sources and their Transform Pairs

\begin{tabular}{c|c|c}
\hline \hline Source & $S(\rho)$ & $\bar{S}(k)$ \\
\hline \hline Point & $\delta(\rho) / 2 \pi \rho$ & 1 \\
N-Ring & $\sum_{i=1}^{N} a_{i} \delta\left(\rho-\rho_{i}\right) / 2 \pi \rho$ & $\sum_{i=1}^{N} a_{i} J_{0}\left(k \rho_{i}\right)$ \\
Disk & $1 / 2 \pi \rho_{0}^{2}$ & $\frac{2}{k \rho_{0}} J_{1}\left(k \rho_{0}\right)$ \\
\hline \hline
\end{tabular}


These integrals are challenging because of their oscillatory nature, involving double Bessel Functions and the semi-infinite range. Williams obtained his results by using a specialized technique. Following inquiry from Williams, We (Loyalka) evaluated these integrals by using Mathematica. We carried out these numerical integrations by three techniques:

1. Highly adaptive schemes that were available as options in Mathematica

2. Segmenting over regions defined by the zeros of the Bessel Functions, we used up to about $\sim 320,000$ segments in one extreme case.

3. Levin's Method [Levin 1996]

These techniques generally confirmed the accuracy (to five figures in almost all cases, although some discrepancies remained in a few rare cases) of the results obtained by Williams and Ganapol and Kornreich. Levin's method appeared very well suited for evaluation of oscillatory integrals of this type, and also allowed very close approach to singularity in the cases of point and ring sources. For the purposes of the present paper, we have recomputed all the results using the Levin's method to a high recursion and precision goal of 7 or 8 , and we have reported our results to six figures in Tables 2 (for the point source, compare with Tables 1a and 2a, respectively of Williams and Ganapol and Kornreich) and 3. We believe our results are accurate to the figures quoted, and when rounded (up) to five figures they agree with all entries in Table 2a of Ganapol and Kornreich. Regarding the ring source, we have also included tables 4 (compare with tables 2 and $2 \mathrm{~b}$ respectively, of Williams and Ganapol and Kornreich) and Table 5 (to show behavior near the singularity). We have reported our results to six figures, believing these to be accurate to this level. On rounding to five figures, our results agree with those of Williams and Ganapol and Kornreich, and at 2.95 agree in the fifth figure with Williams rather than Ganapol and Kornreich. We have not reported results for the disk case, as both Williams and Ganapol and Kornreich are in full agreement. Note that in the values reported below, we have followed, in all tables, the convention of rounding the numbers up.

We further note that the tables 3 and 5 show how well the numerical integrations have performed near the singularities. These also allow an assessment of the nature of the Williams' expressions for the behavior near the singularities, and their accuracy. The seemingly large deviations from the Williams' expressions (in the Tables 3 and 5) are because his results shown 
are those obtained only from his expressions near the singularities, and are not from his full results (this is noted in the Tables 3 and 5). Williams' expressions are not exact, but they describe the behavior near the singularities well.

We also note that the computations were carried out on two different PC's, and while we did not track the computational times, the tables were generated in a few (5-6) hours of CPU time. The calculations can be expedited by using parallel processors (which is straightforward), but the computations of this nature in Mathematica are necessarily time intensive. 
Table 2: Flux for negatively mono-directional point source $($ Nadir) when $\mathbf{c}=0.99$

\begin{tabular}{|c|c|c|c|c|}
\hline$\rho \backslash z_{o}$ & 0.1 & 1 & 2 & 4 \\
\hline 0.1 & $6.19776 \mathrm{E}-01$ & $5.86958 \mathrm{E}-01$ & $2.54786 \mathrm{E}-01$ & 4.81899E-02 \\
\hline 0.2 & $1.87171 \mathrm{E}-01$ & 3.18519E-01 & $1.55446 \mathrm{E}-01$ & 3.46907E-02 \\
\hline 0.3 & 8.73451E-02 & $2.18185 \mathrm{E}-01$ & $1.17598 \mathrm{E}-01$ & $2.94779 \mathrm{E}-02$ \\
\hline 0.4 & 4.99186E-02 & $1.63087 \mathrm{E}-01$ & $9.60956 \mathrm{E}-02$ & $2.64430 \mathrm{E}-02$ \\
\hline 0.5 & 3.20301E-02 & $1.27534 \mathrm{E}-01$ & $8.15517 \mathrm{E}-02$ & $2.43173 \mathrm{E}-02$ \\
\hline 0.6 & $2.21378 \mathrm{E}-02$ & $1.02544 \mathrm{E}-01$ & $7.07279 \mathrm{E}-02$ & $2.26648 \mathrm{E}-02$ \\
\hline 0.7 & 1.61102E-02 & $8.40520 \mathrm{E}-02$ & $6.21899 \mathrm{E}-02$ & $2.12940 \mathrm{E}-02$ \\
\hline 0.8 & $1.21753 \mathrm{E}-02$ & $6.98978 \mathrm{E}-02$ & 5.51961E-02 & $2.01073 \mathrm{E}-02$ \\
\hline 0.9 & $9.47079 \mathrm{E}-03$ & $5.88005 \mathrm{E}-02$ & 4.93191E-02 & $1.90496 \mathrm{E}-02$ \\
\hline 1.0 & $7.53648 \mathrm{E}-03$ & 4.99406E-02 & 4.42914E-02 & 1.80879E-02 \\
\hline 1.1 & 6.10849E-03 & 4.27643E-02 & 3.99344E-02 & $1.72008 \mathrm{E}-02$ \\
\hline 1.2 & $5.02678 \mathrm{E}-03$ & 3.68819E-02 & $3.61225 \mathrm{E}-02$ & 1.63743E-02 \\
\hline 1.3 & 4.18968E-03 & $3.20108 \mathrm{E}-02$ & $3.27632 \mathrm{E}-02$ & $1.55986 \mathrm{E}-02$ \\
\hline 1.4 & $3.53013 \mathrm{E}-03$ & $2.79414 \mathrm{E}-02$ & $2.97861 \mathrm{E}-02$ & 1.48667E-02 \\
\hline 1.5 & $3.00243 \mathrm{E}-03$ & $2.45149 \mathrm{E}-02$ & $2.71358 \mathrm{E}-02$ & 1.41737E-02 \\
\hline 1.6 & $2.57461 \mathrm{E}-03$ & $2.16093 \mathrm{E}-02$ & $2.47677 \mathrm{E}-02$ & $1.35156 \mathrm{E}-02$ \\
\hline 1.7 & $2.22374 \mathrm{E}-03$ & 1.91297E-02 & $2.26452 \mathrm{E}-02$ & $1.28896 \mathrm{E}-02$ \\
\hline 1.8 & $1.93304 \mathrm{E}-03$ & $1.70013 \mathrm{E}-02$ & $2.07378 \mathrm{E}-02$ & 1.22933E-02 \\
\hline 1.9 & $1.69002 \mathrm{E}-03$ & $1.51648 \mathrm{E}-02$ & $1.90196 \mathrm{E}-02$ & $1.17248 \mathrm{E}-02$ \\
\hline 2.0 & $1.48523 \mathrm{E}-03$ & $1.35722 \mathrm{E}-02$ & $1.74687 \mathrm{E}-02$ & $1.11824 \mathrm{E}-02$ \\
\hline 2.1 & $1.31140 \mathrm{E}-03$ & $1.21849 \mathrm{E}-02$ & $1.60661 \mathrm{E}-02$ & $1.06649 \mathrm{E}-02$ \\
\hline 2.2 & 1.16289E-03 & $1.09714 \mathrm{E}-02$ & $1.47954 \mathrm{E}-02$ & 1.01710E-02 \\
\hline 2.3 & $1.03526 \mathrm{E}-03$ & $9.90569 \mathrm{E}-03$ & $1.36423 \mathrm{E}-02$ & 9.69959E-03 \\
\hline 2.4 & $9.24980 \mathrm{E}-04$ & 8.96645E-03 & $1.25943 \mathrm{E}-02$ & 9.24973E-03 \\
\hline 2.5 & 8.29219E-04 & 8.13582E-03 & $1.16404 \mathrm{E}-02$ & $8.82048 \mathrm{E}-03$ \\
\hline 2.6 & $7.45686 \mathrm{E}-04$ & $7.39890 \mathrm{E}-03$ & $1.07710 \mathrm{E}-02$ & $8.41096 \mathrm{E}-03$ \\
\hline 2.7 & $6.72512 \mathrm{E}-04$ & $6.74314 \mathrm{E}-03$ & $9.97747 \mathrm{E}-03$ & 8.02035E-03 \\
\hline 2.8 & 6.08162E-04 & 6.15796E-03 & $9.25226 \mathrm{E}-03$ & 7.64786E-03 \\
\hline 2.9 & $5.51366 \mathrm{E}-04$ & $5.63438 \mathrm{E}-03$ & 8.58867E-03 & 7.29272E-03 \\
\hline 3.0 & $5.01067 \mathrm{E}-04$ & $5.16472 \mathrm{E}-03$ & $7.98070 \mathrm{E}-03$ & $6.95420 \mathrm{E}-03$ \\
\hline 3.1 & $4.56380 \mathrm{E}-04$ & 4.74244E-03 & $7.42305 \mathrm{E}-03$ & $6.63160 \mathrm{E}-03$ \\
\hline 3.2 & $4.16561 \mathrm{E}-04$ & $4.36188 \mathrm{E}-03$ & $6.91095 \mathrm{E}-03$ & $6.32423 \mathrm{E}-03$ \\
\hline 3.3 & $3.80980 \mathrm{E}-04$ & 4.01819E-03 & $6.44016 \mathrm{E}-03$ & $6.03142 \mathrm{E}-03$ \\
\hline 3.4 & $3.49102 \mathrm{E}-04$ & $3.70717 \mathrm{E}-03$ & $6.00686 \mathrm{E}-03$ & $5.75253 \mathrm{E}-03$ \\
\hline 3.5 & $3.20469 \mathrm{E}-04$ & $3.42515 \mathrm{E}-03$ & $5.60766 \mathrm{E}-03$ & $5.48695 \mathrm{E}-03$ \\
\hline 3.6 & 2.94692E-04 & $3.16896 \mathrm{E}-03$ & $5.23948 \mathrm{E}-03$ & $5.23407 \mathrm{E}-03$ \\
\hline 3.7 & 2.71432E-04 & $2.93582 \mathrm{E}-03$ & $4.89958 \mathrm{E}-03$ & 4.99331E-03 \\
\hline 3.8 & $2.50399 \mathrm{E}-04$ & $2.72329 \mathrm{E}-03$ & $4.58546 \mathrm{E}-03$ & $4.76412 \mathrm{E}-03$ \\
\hline 3.9 & $2.31342 \mathrm{E}-04$ & $2.52923 \mathrm{E}-03$ & 4.29489E-03 & 4.54595E-03 \\
\hline 4.0 & $2.14041 \mathrm{E}-04$ & $2.35176 \mathrm{E}-03$ & $4.02586 \mathrm{E}-03$ & $4.33830 \mathrm{E}-03$ \\
\hline
\end{tabular}




\begin{tabular}{l|l|l|l|l}
4.1 & $1.98306 \mathrm{E}-04$ & $2.18922 \mathrm{E}-03$ & $3.77653 \mathrm{E}-03$ & $4.14066 \mathrm{E}-03$ \\
4.2 & $1.83969 \mathrm{E}-04$ & $2.04013 \mathrm{E}-03$ & $3.54526 \mathrm{E}-03$ & $3.95255 \mathrm{E}-03$ \\
4.3 & $1.70884 \mathrm{E}-04$ & $1.90320 \mathrm{E}-03$ & $3.33055 \mathrm{E}-03$ & $3.77352 \mathrm{E}-03$ \\
4.4 & $1.58922 \mathrm{E}-04$ & $1.77726 \mathrm{E}-03$ & $3.13104 \mathrm{E}-03$ & $3.60313 \mathrm{E}-03$ \\
4.5 & $1.47970 \mathrm{E}-04$ & $1.66128 \mathrm{E}-03$ & $2.94549 \mathrm{E}-03$ & $3.44097 \mathrm{E}-03$ \\
4.6 & $1.37927 \mathrm{E}-04$ & $1.55434 \mathrm{E}-03$ & $2.77208 \mathrm{E}-03$ & $3.28661 \mathrm{E}-03$ \\
4.7 & $1.28705 \mathrm{E}-04$ & $1.45562 \mathrm{E}-03$ & $2.61194 \mathrm{E}-03$ & $3.13970 \mathrm{E}-03$ \\
4.8 & $1.20225 \mathrm{E}-04$ & $1.36437 \mathrm{E}-03$ & $2.46199 \mathrm{E}-03$ & $2.99985 \mathrm{E}-03$ \\
4.9 & $1.12417 \mathrm{E}-04$ & $1.27994 \mathrm{E}-03$ & $2.32208 \mathrm{E}-03$ & $2.86672 \mathrm{E}-03$ \\
5.0 & $1.05218 \mathrm{E}-04$ & $1.20173 \mathrm{E}-03$ & $2.19147 \mathrm{E}-03$ & $2.73998 \mathrm{E}-03$ \\
\hline \hline
\end{tabular}


Table 3: Flux for negatively directed mono-directional point source (Nadir) when $c=0.99, z_{0}=0.1$, Approach to the source (singularity). Similar results are obtained for other cases

Williams (limiting results): $\sim \frac{c}{8 \rho} e^{-z_{0}}$.

\begin{tabular}{|c|c|c|}
\hline \hline$\rho$ & Our Results & Williams (limiting results) \\
\hline \hline $1.00 \mathrm{E}-01$ & $6.19776 \mathrm{E}-01$ & $1.11974 \mathrm{E}+00$ \\
$1.00 \mathrm{E}-02$ & $1.08159 \mathrm{E}+01$ & $1.11974 \mathrm{E}+01$ \\
$1.00 \mathrm{E}-03$ & $1.11924 \mathrm{E}+02$ & $1.11974 \mathrm{E}+02$ \\
$1.00 \mathrm{E}-04$ & $1.12218 \mathrm{E}+03$ & $1.11974 \mathrm{E}+03$ \\
$1.00 \mathrm{E}-05$ & $1.12247 \mathrm{E}+04$ & $1.11974 \mathrm{E}+04$ \\
$1.00 \mathrm{E}-06$ & $1.12250 \mathrm{E}+05$ & $1.11974 \mathrm{E}+05$ \\
$1.00 \mathrm{E}-07$ & $1.12250 \mathrm{E}+06$ & $1.11974 \mathrm{E}+06$ \\
$1.00 \mathrm{E}-08$ & $1.12250 \mathrm{E}+07$ & $1.11974 \mathrm{E}+07$ \\
$1.00 \mathrm{E}-09$ & $1.12250 \mathrm{E}+08$ & $1.11974 \mathrm{E}+08$ \\
\hline \hline
\end{tabular}


Table 4: Flux for ring source (Nadir) when $c=0.99, z_{0}=1.0, \rho_{0}=3.0$

\begin{tabular}{|c|c|c|}
\hline \multirow[b]{2}{*}{$\rho$} & \multicolumn{2}{|c|}{$z_{0}=1.0$} \\
\hline & 6 sig. figs. & 5 sig. figs. \\
\hline 0.1 & $5.17285 \mathrm{E}-03$ & $5.1728 \mathrm{E}-03$ \\
\hline 0.2 & $5.19730 \mathrm{E}-03$ & $5.1973 \mathrm{E}-03$ \\
\hline 0.3 & $5.23832 \mathrm{E}-03$ & $5.2383 \mathrm{E}-03$ \\
\hline 0.4 & $5.29636 \mathrm{E}-03$ & $5.2964 \mathrm{E}-03$ \\
\hline 0.5 & $5.37202 \mathrm{E}-03$ & $5.3720 \mathrm{E}-03$ \\
\hline 0.6 & $5.46610 \mathrm{E}-03$ & $5.4661 \mathrm{E}-03$ \\
\hline 0.7 & $5.57963 \mathrm{E}-03$ & $5.5796 \mathrm{E}-03$ \\
\hline 0.8 & $5.71387 \mathrm{E}-03$ & $5.7139 \mathrm{E}-03$ \\
\hline 0.9 & $5.87035 \mathrm{E}-03$ & $5.8704 \mathrm{E}-03$ \\
\hline 1 & $6.05092 \mathrm{E}-03$ & $6.0509 \mathrm{E}-03$ \\
\hline 1.1 & $6.25774 \mathrm{E}-03$ & $6.2577 \mathrm{E}-03$ \\
\hline 1.2 & $6.49343 \mathrm{E}-03$ & $6.4934 \mathrm{E}-03$ \\
\hline 1.3 & $6.76106 \mathrm{E}-03$ & $6.7611 \mathrm{E}-03$ \\
\hline 1.4 & $7.06428 \mathrm{E}-03$ & $7.0643 \mathrm{E}-03$ \\
\hline 1.5 & 7.40739E-03 & 7.4074E-03 \\
\hline 1.6 & 7.79554E-03 & $7.7955 \mathrm{E}-03$ \\
\hline 1.7 & $8.23484 \mathrm{E}-03$ & $8.2348 \mathrm{E}-03$ \\
\hline 1.8 & $8.73262 \mathrm{E}-03$ & $8.7326 \mathrm{E}-03$ \\
\hline 1.9 & $9.29768 \mathrm{E}-03$ & $9.2977 \mathrm{E}-03$ \\
\hline 2 & $9.94070 \mathrm{E}-03$ & $9.9407 \mathrm{E}-03$ \\
\hline 2.1 & $1.06748 \mathrm{E}-02$ & $1.0675 \mathrm{E}-02$ \\
\hline 2.2 & $1.15163 \mathrm{E}-02$ & $1.1516 \mathrm{E}-02$ \\
\hline 2.3 & $1.24859 \mathrm{E}-02$ & $1.2486 \mathrm{E}-02$ \\
\hline 2.4 & $1.36115 \mathrm{E}-02$ & $1.3611 \mathrm{E}-02$ \\
\hline 2.5 & 1.49319E-02 & $1.4932 \mathrm{E}-02$ \\
\hline 2.6 & $1.65080 \mathrm{E}-02$ & $1.6508 \mathrm{E}-02$ \\
\hline 2.7 & $1.84487 \mathrm{E}-02$ & $1.8449 \mathrm{E}-02$ \\
\hline 2.8 & $2.09962 \mathrm{E}-02$ & $2.0996 \mathrm{E}-02$ \\
\hline 2.9 & $2.49381 \mathrm{E}-02$ & $2.4938 \mathrm{E}-02$ \\
\hline 2.91 & $2.55042 \mathrm{E}-02$ & $2.5504 \mathrm{E}-02$ \\
\hline 2.92 & 2.61295E-02 & $2.6130 \mathrm{E}-02$ \\
\hline 2.93 & 2.68299E-02 & $2.6830 \mathrm{E}-02$ \\
\hline 2.94 & $2.76285 \mathrm{E}-02$ & $2.7629 \mathrm{E}-02$ \\
\hline 2.95 & $2.85617 \mathrm{E}-02$ & $2.8562 \mathrm{E}-02$ \\
\hline 2.96 & $2.96902 \mathrm{E}-02$ & $2.9690 \mathrm{E}-02$ \\
\hline 2.97 & $3.11281 \mathrm{E}-02$ & $3.1128 \mathrm{E}-02$ \\
\hline 2.98 & $3.31317 \mathrm{E}-02$ & $3.3132 \mathrm{E}-02$ \\
\hline 2.99 & $3.65198 \mathrm{E}-02$ & $3.6520 \mathrm{E}-02$ \\
\hline 3.01 & 3.63963E-02 & $3.6396 \mathrm{E}-02$ \\
\hline
\end{tabular}




\begin{tabular}{|c|c|c|}
\hline 3.02 & $3.29076 \mathrm{E}-02$ & $3.2908 \mathrm{E}-02$ \\
\hline 3.03 & $3.08124 \mathrm{E}-02$ & 3.0812E-02 \\
\hline 3.04 & 2.92891E-02 & 2.9289E-02 \\
\hline 3.05 & $2.80798 \mathrm{E}-02$ & $2.8080 \mathrm{E}-02$ \\
\hline 3.06 & $2.70696 \mathrm{E}-02$ & $2.7070 \mathrm{E}-02$ \\
\hline 3.07 & 2.61972E-02 & 2.6197E-02 \\
\hline 3.08 & $2.54261 \mathrm{E}-02$ & $2.5426 \mathrm{E}-02$ \\
\hline 3.09 & $2.47327 \mathrm{E}-02$ & 2.4733E-02 \\
\hline 3.1 & $2.41008 \mathrm{E}-02$ & $2.4101 \mathrm{E}-02$ \\
\hline 3.2 & $1.96024 \mathrm{E}-02$ & $1.9602 \mathrm{E}-02$ \\
\hline 3.3 & $1.66330 \mathrm{E}-02$ & $1.6633 \mathrm{E}-02$ \\
\hline 3.4 & $1.43662 \mathrm{E}-02$ & $1.4366 \mathrm{E}-02$ \\
\hline 3.5 & $1.25368 \mathrm{E}-02$ & $1.2537 \mathrm{E}-02$ \\
\hline 3.6 & 1.10192E-02 & $1.1019 \mathrm{E}-02$ \\
\hline 3.7 & $9.74008 \mathrm{E}-03$ & $9.7401 \mathrm{E}-03$ \\
\hline 3.8 & $8.65053 \mathrm{E}-03$ & $8.6505 \mathrm{E}-03$ \\
\hline 3.9 & 7.71517E-03 & 7.7152E-03 \\
\hline 4 & $6.90711 \mathrm{E}-03$ & $6.9071 \mathrm{E}-03$ \\
\hline 4.1 & $6.20522 \mathrm{E}-03$ & $6.2052 \mathrm{E}-03$ \\
\hline 4.2 & $5.59260 \mathrm{E}-03$ & $5.5926 \mathrm{E}-03$ \\
\hline 4.3 & $5.05554 \mathrm{E}-03$ & $5.0555 \mathrm{E}-03$ \\
\hline 4.4 & $4.58282 \mathrm{E}-03$ & $4.5828 \mathrm{E}-03$ \\
\hline 4.5 & 4.16513E-03 & $4.1651 \mathrm{E}-03$ \\
\hline 4.6 & 3.79477E-03 & $3.7948 \mathrm{E}-03$ \\
\hline 4.7 & $3.46528 \mathrm{E}-03$ & $3.4653 \mathrm{E}-03$ \\
\hline 4.8 & $3.17124 \mathrm{E}-03$ & $3.1712 \mathrm{E}-03$ \\
\hline 4.9 & $2.90807 \mathrm{E}-03$ & $2.9081 \mathrm{E}-03$ \\
\hline 5 & $2.67187 \mathrm{E}-03$ & $2.6719 \mathrm{E}-03$ \\
\hline
\end{tabular}


Table 5: Flux for negatively directed mono-directional ring source (Nadir) when $c=0.99, z_{0}=1.0, \rho_{0}=3.0$ (details as one approaches the source (singularity)

Note that for Table 5, Williams results correspond to only the singular part of the limiting results, or $\sim \frac{c}{8 \pi \rho_{0}} e^{-z_{0}} \log \left(\frac{1}{\left|\rho-\rho_{0}\right|}\right)$

\begin{tabular}{c|c|c}
\hline \hline$\rho$ & $\begin{array}{l}\text { Our } \\
\text { Results }\end{array}$ & Williams \\
\hline \hline 2.90000000000 & 0.0249381 & 0.0111223 \\
2.99000000000 & 0.0365198 & 0.0222446 \\
2.99900000000 & 0.0476674 & 0.033367 \\
2.99990000000 & 0.0588108 & 0.0444893 \\
2.99999000000 & 0.0699597 & 0.0556116 \\
2.99999900000 & 0.0811094 & 0.0667339 \\
2.99999990000 & 0.0922592 & 0.0778562 \\
2.99999999000 & 0.103409 & 0.0889785 \\
2.99999999900 & 0.114559 & 0.100101 \\
2.99999999990 & 0.125709 & 0.111223 \\
3.00000000000 & - & Infinity \\
3.00000000010 & 0.125709 & 0.111223 \\
3.00000000100 & 0.114559 & 0.100101 \\
3.00000001000 & 0.103409 & 0.0889785 \\
3.00000010000 & 0.0922592 & 0.0778562 \\
3.00000100000 & 0.0811094 & 0.0667339 \\
3.00001000000 & 0.0699595 & 0.0556116 \\
3.00010000000 & 0.0588088 & 0.0444893 \\
3.00100000000 & 0.0476513 & 0.033367 \\
3.01000000000 & 0.0363963 & 0.0222446 \\
3.10000000000 & 0.0241008 & 0.0111223 \\
\hline \hline
\end{tabular}




\section{Verification of MCNP-5}

MCNP-5 is well known Monte Carlo code, and as explained by Brown and Barnett, it can be used for one group calculations in a straightforward manner. We generated an appropriate cross-section file, and modified it for different values of $\mathrm{c}$ as we considered different cases. We first carried out some criticality calculations for an infinite bare slab and a bare sphere [Naz and Loyalka, 2007] and also the line source problem [Loyalka and Williams, 2008] to verify against some known accurate results, and then we carried out calculations for point, disc and ring sources. We have not reported the criticality and line source results here (the agreements with MCNP-5 were very good), but include only the point, disc and ring results.

\subsection{Point source}

The infinite bare slab with finite slab thickness (b) was modeled and a mono-directional point source was put on the z-axis. The c value of the medium was set to be 0.99 . The calculation was performed for two different source directions: 1) negatively mono-directional point source (nadir) and 2) positively mono-directional point source (zenith). Also, four different source positions $\left(\mathrm{z}_{0}=0.1,1.0,2.0\right.$ and 4.0) were investigated. The particle density (flux) was calculated on the surface $(\mathrm{z}=0)$ with various radius $(\rho=0,0.1,0.5,1.0,1.5,2.0,2.5,3.0,3.5,4.0$, 4.5 and 5.0). The F5 detector tally was used for this calculation with detector radius of 0.01 and the number of particles simulated(nps) was $1.0 \times 10^{8}$ for all cases.

As the slab thickness (b) increased from 10 to 100, the MCNP calculation results approached the analytic values. However, the results for $b=100$ and $b=1000$ were identical. Table 6 and 7 show the calculation results for nadir and zenith case, respectively, for $b=1000$. The MCNP results agreed well with the analytic results. For most cases the difference between MCNP and analytic result was less than $0.1 \%$. Figure 1 shows the comparison between nadir and zenith results. The nadir case shows a peak at the center of the slab whereas it is relatively flat for the zenith case. 
Table 6: Flux for negatively directed mono-directional point source (Nadir) when c=0.99.

\begin{tabular}{|c|c|c|c|c|c|c|c|c|c|c|c|c|}
\hline \multirow[b]{2}{*}{$\rho$} & \multicolumn{3}{|c|}{$\mathrm{z}_{\mathrm{s}}=0.1$} & \multicolumn{3}{|c|}{$\mathrm{z}_{\mathrm{s}}=1.0$} & \multicolumn{3}{|c|}{$\mathrm{z}_{\mathrm{s}}=2.0$} & \multicolumn{3}{|c|}{$\mathrm{z}_{\mathrm{s}}=4.0$} \\
\hline & $\begin{array}{c}\text { MCNP } \\
(b=1000)\end{array}$ & $\begin{array}{l}\text { Analytic } \\
(b=\infty)\end{array}$ & $\begin{array}{c}\text { Error } \\
(\%)\end{array}$ & $\begin{array}{c}\text { MCNP } \\
(b=1000)\end{array}$ & $\begin{array}{l}\text { Analytic } \\
(b=\infty)\end{array}$ & $\begin{array}{c}\text { Error } \\
(\%)\end{array}$ & $\begin{array}{c}\text { MCNP } \\
(b=1000)\end{array}$ & $\begin{array}{l}\text { Analytic } \\
(b=\infty)\end{array}$ & $\begin{array}{c}\text { Error } \\
(\%)\end{array}$ & $\begin{array}{c}\text { MCNP } \\
(b=1000)\end{array}$ & $\begin{array}{l}\text { Analytic } \\
(b=\infty)\end{array}$ & $\begin{array}{l}\text { Error } \\
(\%)\end{array}$ \\
\hline 0.0 & $2.8284 \mathrm{E}+01$ & $\infty$ & - & $1.1922 \mathrm{E}+01$ & $\infty$ & - & $4.4431 \mathrm{E}+00$ & $\infty$ & - & 6.1436E-01 & 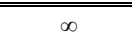 & - \\
\hline 0.1 & $6.1978 \mathrm{E}-01$ & $6.1978 \mathrm{E}-01$ & 0.00 & $5.8694 \mathrm{E}-01$ & $5.8696 \mathrm{E}-01$ & 0.00 & $2.5508 \mathrm{E}-01$ & $2.5478 \mathrm{E}-01$ & 0.12 & 4.8173E-02 & $4.8190 \mathrm{E}-02$ & -0.04 \\
\hline 0.5 & $3.2034 \mathrm{E}-02$ & $3.2030 \mathrm{E}-02$ & 0.01 & $1.2752 \mathrm{E}-01$ & $1.2753 \mathrm{E}-01$ & -0.01 & $8.1579 \mathrm{E}-02$ & $8.1552 \mathrm{E}-02$ & 0.03 & $2.4330 \mathrm{E}-02$ & $2.4317 \mathrm{E}-02$ & 0.05 \\
\hline 1.0 & $7.5434 \mathrm{E}-03$ & $7.5365 \mathrm{E}-03$ & 0.09 & $4.9921 \mathrm{E}-02$ & $4.9941 \mathrm{E}-02$ & -0.04 & $4.4295 \mathrm{E}-02$ & $4.4292 \mathrm{E}-02$ & 0.01 & $1.8085 \mathrm{E}-02$ & $1.8088 \mathrm{E}-02$ & -0.02 \\
\hline 1.5 & $3.0012 \mathrm{E}-03$ & $3.0024 \mathrm{E}-03$ & -0.04 & $2.4513 \mathrm{E}-02$ & $2.4515 \mathrm{E}-02$ & -0.01 & $2.7131 \mathrm{E}-02$ & $2.7136 \mathrm{E}-02$ & -0.02 & $1.4177 \mathrm{E}-02$ & $1.4174 \mathrm{E}-02$ & 0.02 \\
\hline 2.0 & $1.4860 \mathrm{E}-03$ & $1.4851 \mathrm{E}-03$ & 0.06 & $1.3574 \mathrm{E}-02$ & $1.3572 \mathrm{E}-02$ & 0.01 & $1.7466 \mathrm{E}-02$ & $1.7469 \mathrm{E}-02$ & -0.02 & $1.1185 \mathrm{E}-02$ & $1.1182 \mathrm{E}-02$ & 0.03 \\
\hline 2.5 & $8.2742 \mathrm{E}-04$ & $8.2922 \mathrm{E}-04$ & -0.22 & $8.1397 \mathrm{E}-03$ & $8.1358 \mathrm{E}-03$ & 0.05 & $1.1636 \mathrm{E}-02$ & $1.1640 \mathrm{E}-02$ & -0.03 & $8.8237 \mathrm{E}-03$ & $8.8205 \mathrm{E}-03$ & 0.04 \\
\hline 3.0 & 5.0213E-04 & $5.0107 \mathrm{E}-04$ & 0.21 & $5.1670 \mathrm{E}-03$ & $5.1647 \mathrm{E}-03$ & 0.04 & 7.9779E-03 & $7.9807 \mathrm{E}-03$ & -0.04 & $6.9523 \mathrm{E}-03$ & $6.9542 \mathrm{E}-03$ & -0.03 \\
\hline 3.5 & $3.2089 \mathrm{E}-04$ & $3.2047 \mathrm{E}-04$ & 0.13 & $3.4238 \mathrm{E}-03$ & $3.4252 \mathrm{E}-03$ & -0.04 & 5.6037E-03 & $5.6077 \mathrm{E}-03$ & -0.07 & $5.4869 \mathrm{E}-03$ & $5.4869 \mathrm{E}-03$ & 0.00 \\
\hline 4.0 & $2.1443 \mathrm{E}-04$ & $2.1404 \mathrm{E}-04$ & 0.18 & $2.3526 \mathrm{E}-03$ & $2.3518 \mathrm{E}-03$ & 0.03 & $4.0261 \mathrm{E}-03$ & $4.0259 \mathrm{E}-03$ & 0.00 & $4.3398 \mathrm{E}-03$ & $4.3383 \mathrm{E}-03$ & 0.03 \\
\hline 4.5 & $1.4844 \mathrm{E}-04$ & $1.4797 \mathrm{E}-04$ & 0.32 & $1.6627 \mathrm{E}-03$ & $1.6613 \mathrm{E}-03$ & 0.08 & $2.9453 \mathrm{E}-03$ & $2.9455 \mathrm{E}-03$ & -0.01 & $3.4397 \mathrm{E}-03$ & $3.4410 \mathrm{E}-03$ & -0.04 \\
\hline 5.0 & $1.0521 \mathrm{E}-04$ & $1.0522 \mathrm{E}-04$ & -0.01 & $1.2024 \mathrm{E}-03$ & $1.2017 \mathrm{E}-03$ & 0.06 & $2.1915 \mathrm{E}-03$ & $2.1915 \mathrm{E}-03$ & 0.00 & $2.7412 \mathrm{E}-03$ & $2.7400 \mathrm{E}-03$ & 0.04 \\
\hline
\end{tabular}


Table 7: Flux for positively directed mono-directional point source (Zenith) when $\mathrm{c}=0.99$.

\begin{tabular}{|c|c|c|c|c|c|c|c|c|c|c|c|c|}
\hline \multirow[b]{2}{*}{$\rho$} & \multicolumn{3}{|c|}{$\mathrm{z}_{\mathrm{s}}=0.1$} & \multicolumn{3}{|c|}{$\mathrm{z}_{\mathrm{s}}=1.0$} & \multicolumn{3}{|c|}{$\mathrm{z}_{\mathrm{s}}=2.0$} & \multicolumn{3}{|c|}{$\mathrm{z}_{\mathrm{s}}=4.0$} \\
\hline & $\begin{array}{c}\text { MCNP } \\
(b=1000)\end{array}$ & $\begin{array}{l}\text { Analytic } \\
(b=\infty)\end{array}$ & $\begin{array}{l}\text { Error } \\
(\%)\end{array}$ & $\begin{array}{c}\text { MCNP } \\
(b=1000)\end{array}$ & $\begin{array}{l}\text { Analytic } \\
(b=\infty)\end{array}$ & $\begin{array}{c}\text { Error } \\
(\%)\end{array}$ & $\begin{array}{c}\text { MCNP } \\
(b=1000)\end{array}$ & $\begin{array}{l}\text { Analytic } \\
(b=\infty)\end{array}$ & $\begin{array}{l}\text { Error } \\
(\%)\end{array}$ & $\begin{array}{c}\text { MCNP } \\
(b=1000)\end{array}$ & $\begin{array}{l}\text { Analytic } \\
(b=\infty)\end{array}$ & $\begin{array}{l}\text { Error } \\
(\%)\end{array}$ \\
\hline 0.0 & $7.4128 \mathrm{E}-01$ & $7.4060 \mathrm{E}-01$ & $0.09 \%$ & $5.5406 \mathrm{E}-02$ & 5.5435E-02 & $-0.05 \%$ & $2.1442 \mathrm{E}-02$ & $2.1538 \mathrm{E}-02$ & $-0.44 \%$ & $6.8389 \mathrm{E}-03$ & $6.8500 \mathrm{E}-03$ & $-0.16 \%$ \\
\hline 0.1 & $5.6669 \mathrm{E}-01$ & $5.6660 \mathrm{E}-01$ & $0.02 \%$ & $5.5156 \mathrm{E}-02$ & $5.5125 \mathrm{E}-02$ & $0.06 \%$ & $2.1480 \mathrm{E}-02$ & $2.1498 \mathrm{E}-02$ & $-0.08 \%$ & $6.8486 \mathrm{E}-03$ & $6.8456 \mathrm{E}-03$ & $0.04 \%$ \\
\hline 0.5 & $1.5823 \mathrm{E}-01$ & $1.5823 \mathrm{E}-01$ & $0.00 \%$ & $4.8669 \mathrm{E}-02$ & $4.8705 \mathrm{E}-02$ & $-0.07 \%$ & $2.0576 \mathrm{E}-02$ & $2.0588 \mathrm{E}-02$ & $-0.06 \%$ & $6.7415 \mathrm{E}-03$ & $6.7423 \mathrm{E}-03$ & $-0.01 \%$ \\
\hline 1.0 & $6.3915 \mathrm{E}-02$ & $6.3921 \mathrm{E}-02$ & $-0.01 \%$ & $3.6008 \mathrm{E}-02$ & $3.6028 \mathrm{E}-02$ & $-0.06 \%$ & $1.8159 \mathrm{E}-02$ & $1.8161 \mathrm{E}-02$ & $-0.01 \%$ & $6.4355 \mathrm{E}-03$ & $6.4352 \mathrm{E}-03$ & $0.00 \%$ \\
\hline 1.5 & $3.3350 \mathrm{E}-02$ & $3.3359 \mathrm{E}-02$ & $-0.03 \%$ & $2.5197 \mathrm{E}-02$ & $2.5198 \mathrm{E}-02$ & $0.00 \%$ & $1.5122 \mathrm{E}-02$ & $1.5118 \mathrm{E}-02$ & $0.03 \%$ & $5.9675 \mathrm{E}-03$ & $5.9717 \mathrm{E}-03$ & $-0.07 \%$ \\
\hline 2.0 & $1.9620 \mathrm{E}-02$ & $1.9624 \mathrm{E}-02$ & $-0.02 \%$ & $1.7553 \mathrm{E}-02$ & $1.7550 \mathrm{E}-02$ & $0.02 \%$ & $1.2154 \mathrm{E}-02$ & $1.2146 \mathrm{E}-02$ & $0.07 \%$ & $5.4082 \mathrm{E}-03$ & $5.4078 \mathrm{E}-03$ & $0.01 \%$ \\
\hline 2.5 & $1.2403 \mathrm{E}-02$ & $1.2405 \mathrm{E}-02$ & $-0.02 \%$ & $1.2370 \mathrm{E}-02$ & $1.2373 \mathrm{E}-02$ & $-0.02 \%$ & $9.5790 \mathrm{E}-03$ & $9.5775 \mathrm{E}-03$ & $0.02 \%$ & $4.7946 \mathrm{E}-03$ & 4.7992E-03 & $-0.10 \%$ \\
\hline 3.0 & $8.2424 \mathrm{E}-03$ & $8.2402 \mathrm{E}-03$ & $0.03 \%$ & $8.8633 \mathrm{E}-03$ & $8.8688 \mathrm{E}-03$ & $-0.06 \%$ & $7.4931 \mathrm{E}-03$ & $7.4910 \mathrm{E}-03$ & $0.03 \%$ & $4.1922 \mathrm{E}-03$ & 4.1913E-03 & $0.02 \%$ \\
\hline 3.5 & $5.6776 \mathrm{E}-03$ & $5.6792 \mathrm{E}-03$ & $-0.03 \%$ & $6.4643 \mathrm{E}-03$ & $6.4641 \mathrm{E}-03$ & $0.00 \%$ & $5.8486 \mathrm{E}-03$ & $5.8492 \mathrm{E}-03$ & $-0.01 \%$ & $3.6153 \mathrm{E}-03$ & $3.6157 \mathrm{E}-03$ & $-0.01 \%$ \\
\hline 4.0 & $4.0326 \mathrm{E}-03$ & $4.0294 \mathrm{E}-03$ & $0.08 \%$ & $4.7869 \mathrm{E}-03$ & $4.7862 \mathrm{E}-03$ & $0.01 \%$ & $4.5758 \mathrm{E}-03$ & $4.5763 \mathrm{E}-03$ & $-0.01 \%$ & $3.0914 \mathrm{E}-03$ & $3.0909 \mathrm{E}-03$ & $0.02 \%$ \\
\hline 4.5 & $2.9273 \mathrm{E}-03$ & $2.9274 \mathrm{E}-03$ & $0.00 \%$ & $3.5946 \mathrm{E}-03$ & $3.5954 \mathrm{E}-03$ & $-0.02 \%$ & $3.5948 \mathrm{E}-03$ & $3.5949 \mathrm{E}-03$ & $0.00 \%$ & $2.6250 \mathrm{E}-03$ & $2.6253 \mathrm{E}-03$ & $-0.01 \%$ \\
\hline 5.0 & $2.1728 \mathrm{E}-03$ & $2.1692 \mathrm{E}-03$ & $0.17 \%$ & $2.7385 \mathrm{E}-03$ & $2.7365 \mathrm{E}-03$ & $0.07 \%$ & $2.8400 \mathrm{E}-03$ & $2.8386 \mathrm{E}-03$ & $0.05 \%$ & $2.2198 \mathrm{E}-03$ & $2.2202 \mathrm{E}-03$ & $-0.02 \%$ \\
\hline
\end{tabular}




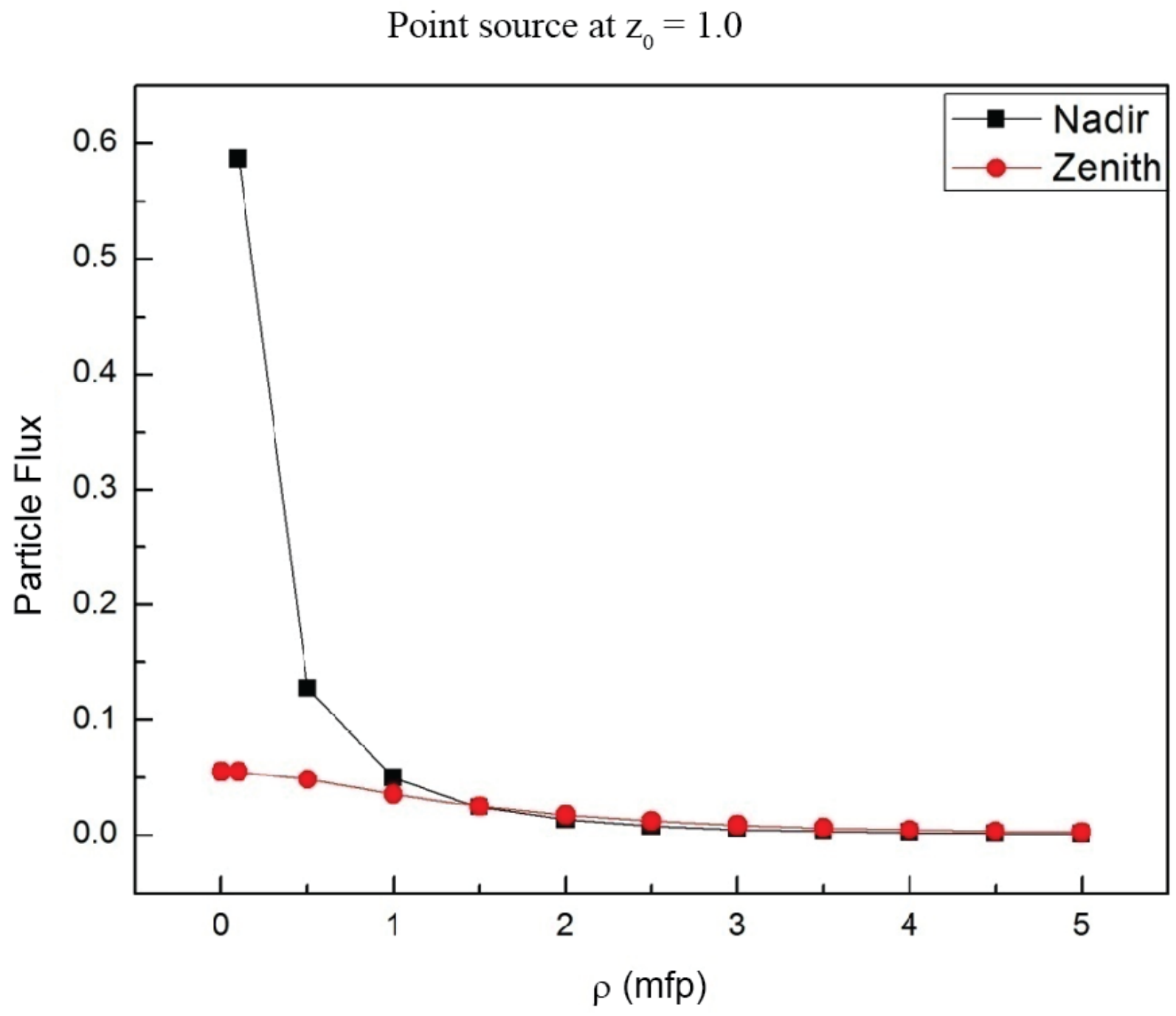

Figure 1: Particle density (flux) due to a point source for different source directions when c=0.99. 
We carried out MCNP calculations for the zenith case with several different detector sizes $(0.1,0.01$, and 0.001) to find the effect of detector size on the result was explored. Figure 2 shows the ratio of MCNP result to analytic result for $z_{\mathrm{s}}=0.1,1.0,2.0$ and 1.0. When the detector size was decreased from 0.1 to 0.01 , the MCNP result showed better agreement with the analytic result. However, when the detector size was decreased further to 0.001 , the result were about the same. 

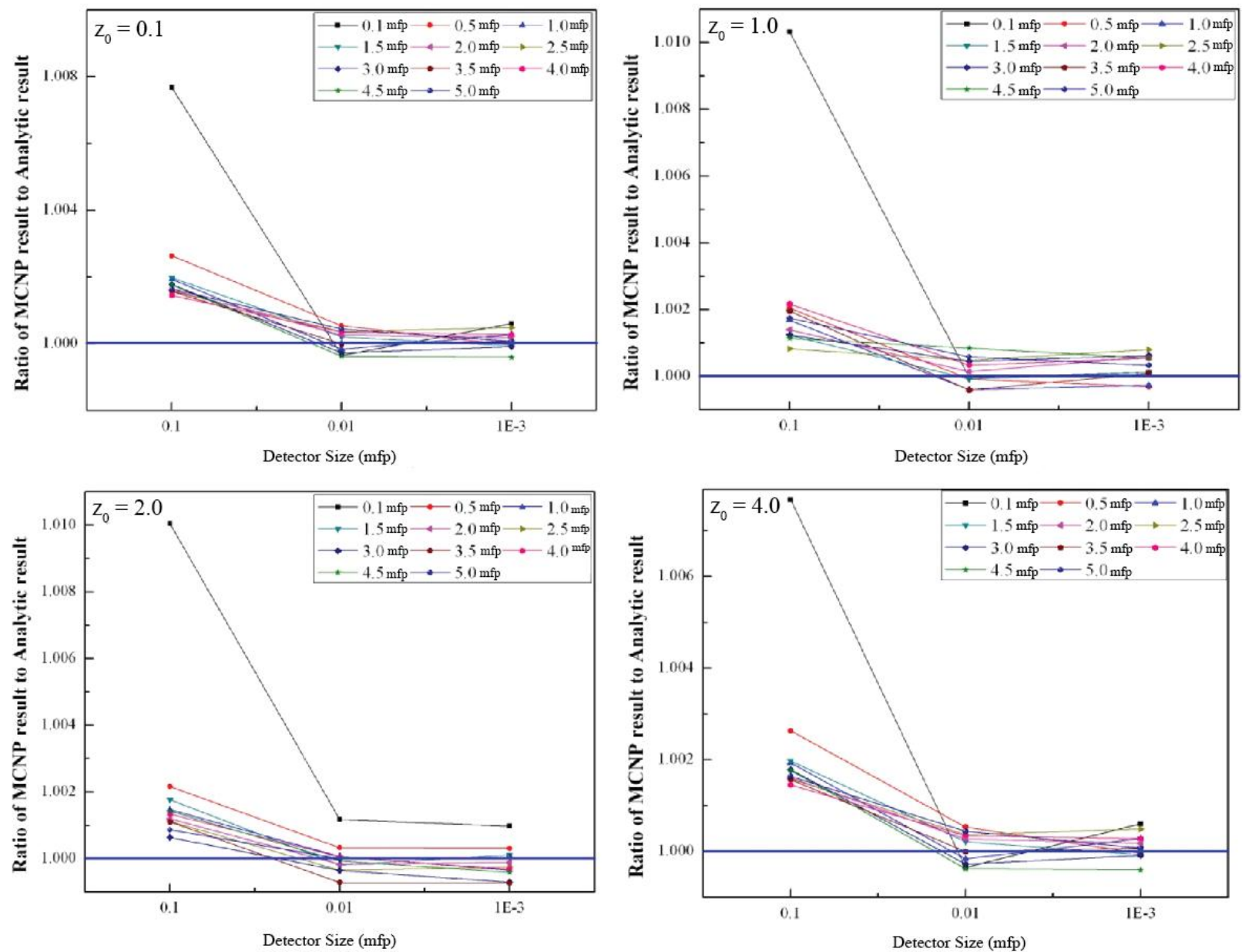

Figure 2: The ratio of MCNP result to analytic result for the comparison of the different detector sizes.

MCNP calculations were also performed for the zenith case with several different numbers of particle simulated to find the effect of simulation size on the result. Figure 6 shows the ratio of MCNP result to analytic 
result for $\mathrm{z}_{\mathrm{s}}=0.1$. As the number of particles simulated was increased by $10^{8}$, the MCNP result showed better agreement with the analytic result as expected. 


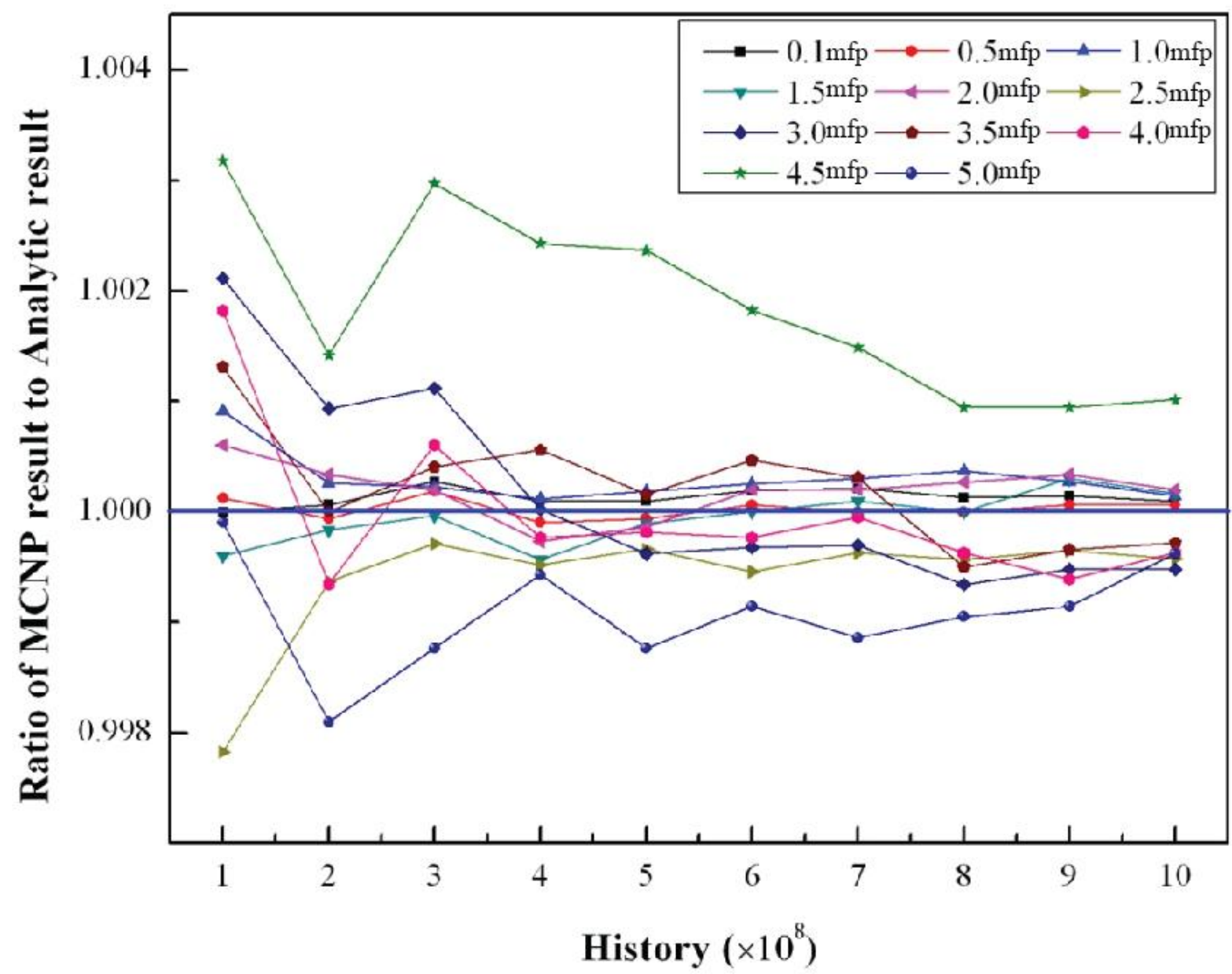

Figure 3: The ratio of MCNP result to analytic result for the comparison of different histories (nps). 
Although no analytical results have been reported for this case, for future comparisons, we carried out some calculations for an isotropic point source instead of mono-directional point source. For both $\mathrm{c}=0.99$ and 1.0 the particle density ((flux) was calculated when the source was located at $z_{\mathrm{s}}=0.1$. This calculation was performed by the use of a F5 tally with detector radius of 0.1 . The number of particles simulated was $10^{8}$ except for the case of $\mathrm{c}=1.0$ and $b=1000$ for the computing time. The result is shown in table 10 . 
Table 8: Flux for isotropic point source at $\mathrm{z}_{0}=0.1 \mathrm{mfp}$.

\begin{tabular}{c|cc|cc|cc|cc}
\hline \hline \multirow{2}{*}{$\rho$} & \multicolumn{2}{|c|}{$\mathrm{b}=1$} & \multicolumn{2}{|c|}{$\mathrm{b}=10$} & \multicolumn{2}{c|}{$\mathrm{b}=100$} & \multicolumn{2}{c}{$\mathrm{b}=1000$} \\
\cline { 2 - 8 } & $\mathrm{c}=0.99$ & $\mathrm{c}=1.0$ & $\mathrm{c}=0.99$ & $\mathrm{c}=1.0$ & $\mathrm{c}=0.99$ & $\mathrm{c}=1.0$ & $\mathrm{c}=0.99$ & $\mathrm{c}=1.0$ \\
\hline \hline 0.0 & $8.8489 \mathrm{E}+00$ & $8.8691 \mathrm{E}+00$ & $8.8589 \mathrm{E}+00$ & $8.8800 \mathrm{E}+00$ & $8.8589 \mathrm{E}+00$ & $8.8801 \mathrm{E}+00$ & $8.8589 \mathrm{E}+00$ & $8.8803 \mathrm{E}+00$ \\
0.1 & $4.3995 \mathrm{E}+00$ & $4.4119 \mathrm{E}+00$ & $4.4094 \mathrm{E}+00$ & $4.4229 \mathrm{E}+00$ & $4.4094 \mathrm{E}+00$ & $4.4230 \mathrm{E}+00$ & $4.4094 \mathrm{E}+00$ & $4.4231 \mathrm{E}+00$ \\
0.5 & $3.3952 \mathrm{E}-01$ & $3.4238 \mathrm{E}-01$ & $3.4900 \mathrm{E}-01$ & $3.5285 \mathrm{E}-01$ & $3.4902 \mathrm{E}-01$ & $3.5293 \mathrm{E}-01$ & $3.4902 \mathrm{E}-01$ & $3.5292 \mathrm{E}-01$ \\
1.0 & $7.6495 \mathrm{E}-02$ & $7.7609 \mathrm{E}-02$ & $8.4813 \mathrm{E}-02$ & $8.6840 \mathrm{E}-02$ & $8.4830 \mathrm{E}-02$ & $8.6921 \mathrm{E}-02$ & $8.4830 \mathrm{E}-02$ & $8.6919 \mathrm{E}-02$ \\
1.5 & $2.7319 \mathrm{E}-02$ & $2.7854 \mathrm{E}-02$ & $3.4164 \mathrm{E}-02$ & $3.5514 \mathrm{E}-02$ & $3.4181 \mathrm{E}-02$ & $3.5594 \mathrm{E}-02$ & $3.4181 \mathrm{E}-02$ & $3.5585 \mathrm{E}-02$ \\
2.0 & $1.1615 \mathrm{E}-02$ & $1.1891 \mathrm{E}-02$ & $1.7006 \mathrm{E}-02$ & $1.7990 \mathrm{E}-02$ & $1.7022 \mathrm{E}-02$ & $1.8071 \mathrm{E}-02$ & $1.7022 \mathrm{E}-02$ & $1.8062 \mathrm{E}-02$ \\
2.5 & $5.3858 \mathrm{E}-03$ & $5.5334 \mathrm{E}-03$ & $9.5307 \mathrm{E}-03$ & $1.0284 \mathrm{E}-02$ & $9.5469 \mathrm{E}-03$ & $1.0362 \mathrm{E}-02$ & $9.5469 \mathrm{E}-03$ & $1.0361 \mathrm{E}-02$ \\
3.0 & $2.6332 \mathrm{E}-03$ & $2.7141 \mathrm{E}-03$ & $5.7740 \mathrm{E}-03$ & $6.3702 \mathrm{E}-03$ & $5.7896 \mathrm{E}-03$ & $6.4482 \mathrm{E}-03$ & $5.7896 \mathrm{E}-03$ & $6.4490 \mathrm{E}-03$ \\
3.5 & $1.3331 \mathrm{E}-03$ & $1.3778 \mathrm{E}-03$ & $3.6972 \mathrm{E}-03$ & $4.1779 \mathrm{E}-03$ & $3.7124 \mathrm{E}-03$ & $4.2548 \mathrm{E}-03$ & $3.7124 \mathrm{E}-03$ & $4.2539 \mathrm{E}-03$ \\
4.0 & $6.9079 \mathrm{E}-04$ & $7.1614 \mathrm{E}-04$ & $2.4707 \mathrm{E}-03$ & $2.8649 \mathrm{E}-03$ & $2.4858 \mathrm{E}-03$ & $2.9396 \mathrm{E}-03$ & $2.4858 \mathrm{E}-03$ & $2.9397 \mathrm{E}-03$ \\
4.5 & $3.6507 \mathrm{E}-04$ & $3.7912 \mathrm{E}-04$ & $1.7066 \mathrm{E}-03$ & $2.0339 \mathrm{E}-03$ & $1.7212 \mathrm{E}-03$ & $2.1071 \mathrm{E}-03$ & $1.7212 \mathrm{E}-03$ & $2.1051 \mathrm{E}-03$ \\
5.0 & $1.9525 \mathrm{E}-04$ & $2.0335 \mathrm{E}-04$ & $1.2108 \mathrm{E}-03$ & $1.4850 \mathrm{E}-03$ & $1.2249 \mathrm{E}-03$ & $1.5569 \mathrm{E}-03$ & $1.2249 \mathrm{E}-03$ & $1.5563 \mathrm{E}-03$ \\
\hline \hline
\end{tabular}




\subsection{Ring source}

An infinite bare slab with slab thickness of $b=1000 \mathrm{mfp}$ was modeled and a mono-directional ring source with radius of $3.0 \mathrm{mfp}$ was put at $\mathrm{z}=1 \mathrm{mfp}$ around the $\mathrm{z}$-axis. The $\mathrm{c}$ value of the medium was set to be 0.99 . The calculation was performed for nadir and zenith cases. The particle density was calculated on the surface ( $\mathrm{z}=0)$ with various radius $(\rho=0,0.1,0.5,1.0,1.5,2.0,2.5,3.0,3.5,4.0,4.5$ and 5.0$)$. The F5 detector tally was used for this calculation with detector radius of 0.01 and the number of particles simulated (nps) was $1.0 \times 10^{8}$ for all cases. Table 9 shows the calculation results for both nadir and zenith case. MCNP results agreed well with the analytic results within $1 \%$. Figure 7 shows the neutron density for both nadir and zenith cases for the comparison. The nadir case shows a peak at $\rho=3$, whereas result of the zenith case was relatively flat. 
Table 9: Flux for mono-directional ring source (both nadir and zenith) when $\mathbf{c}=0.99$.

\begin{tabular}{|c|c|c|c|c|c|c|}
\hline \multirow{2}{*}{$\rho$} & \multicolumn{3}{|c|}{ Nadir } & \multicolumn{3}{|c|}{ Zenith } \\
\hline & $\operatorname{MCNP}(b=1000)$ & Analytic $(b=\infty)$ & Error $(\%)$ & $\operatorname{MCNP}(b=1000)$ & Analytic $(b=\infty)$ & Error $(\%)$ \\
\hline 0.0 & 5.1541E-03 & $\begin{array}{l}5.1647 \mathrm{E}-03 \\
\end{array}$ & -0.21 & $8.9051 \mathrm{E}-03$ & - & - \\
\hline 0.1 & $5.1716 \mathrm{E}-03$ & $5.1728 \mathrm{E}-03$ & -0.02 & $8.8798 \mathrm{E}-03$ & $8.8748 \mathrm{E}-03$ & $0.06 \%$ \\
\hline 0.5 & $5.3624 \mathrm{E}-03$ & $5.3720 \mathrm{E}-03$ & -0.18 & $9.0194 \mathrm{E}-03$ & $9.0197 \mathrm{E}-03$ & $0.00 \%$ \\
\hline 1.0 & $6.0491 \mathrm{E}-03$ & $6.0509 \mathrm{E}-03$ & -0.03 & $9.4712 \mathrm{E}-03$ & $9.4712 \mathrm{E}-03$ & $0.00 \%$ \\
\hline 1.5 & 7.4006E-03 & $7.4074 \mathrm{E}-03$ & -0.09 & $1.0196 \mathrm{E}-02$ & $1.0204 \mathrm{E}-02$ & $-0.08 \%$ \\
\hline 2.0 & $9.9382 \mathrm{E}-03$ & $9.9407 \mathrm{E}-03$ & -0.03 & $1.1110 \mathrm{E}-02$ & $1.1110 \mathrm{E}-02$ & $0.00 \%$ \\
\hline 2.5 & $1.4926 \mathrm{E}-02$ & $1.4932 \mathrm{E}-02$ & -0.04 & $1.1820 \mathrm{E}-02$ & $1.1821 \mathrm{E}-02$ & $-0.01 \%$ \\
\hline 3.0 & 4.3753E-02 & - & - & $1.1546 \mathrm{E}-02$ & $1.1445 \mathrm{E}-02$ & $0.88 \%$ \\
\hline 3.5 & $1.2542 \mathrm{E}-02$ & $1.2537 \mathrm{E}-02$ & 0.04 & $9.9231 \mathrm{E}-03$ & $9.9244 \mathrm{E}-03$ & $-0.01 \%$ \\
\hline 4.0 & $6.8998 \mathrm{E}-03$ & $6.9071 \mathrm{E}-03$ & -0.11 & $7.7701 \mathrm{E}-03$ & $7.7729 \mathrm{E}-03$ & $-0.04 \%$ \\
\hline 4.5 & $4.1631 \mathrm{E}-03$ & $4.1651 \mathrm{E}-03$ & -0.05 & $5.8719 \mathrm{E}-03$ & $5.8694 \mathrm{E}-03$ & $0.04 \%$ \\
\hline 5.0 & $2.6735 \mathrm{E}-03$ & $2.6719 \mathrm{E}-03$ & 0.06 & 4.4001E-03 & 4.4007E-03 & $-0.01 \%$ \\
\hline
\end{tabular}




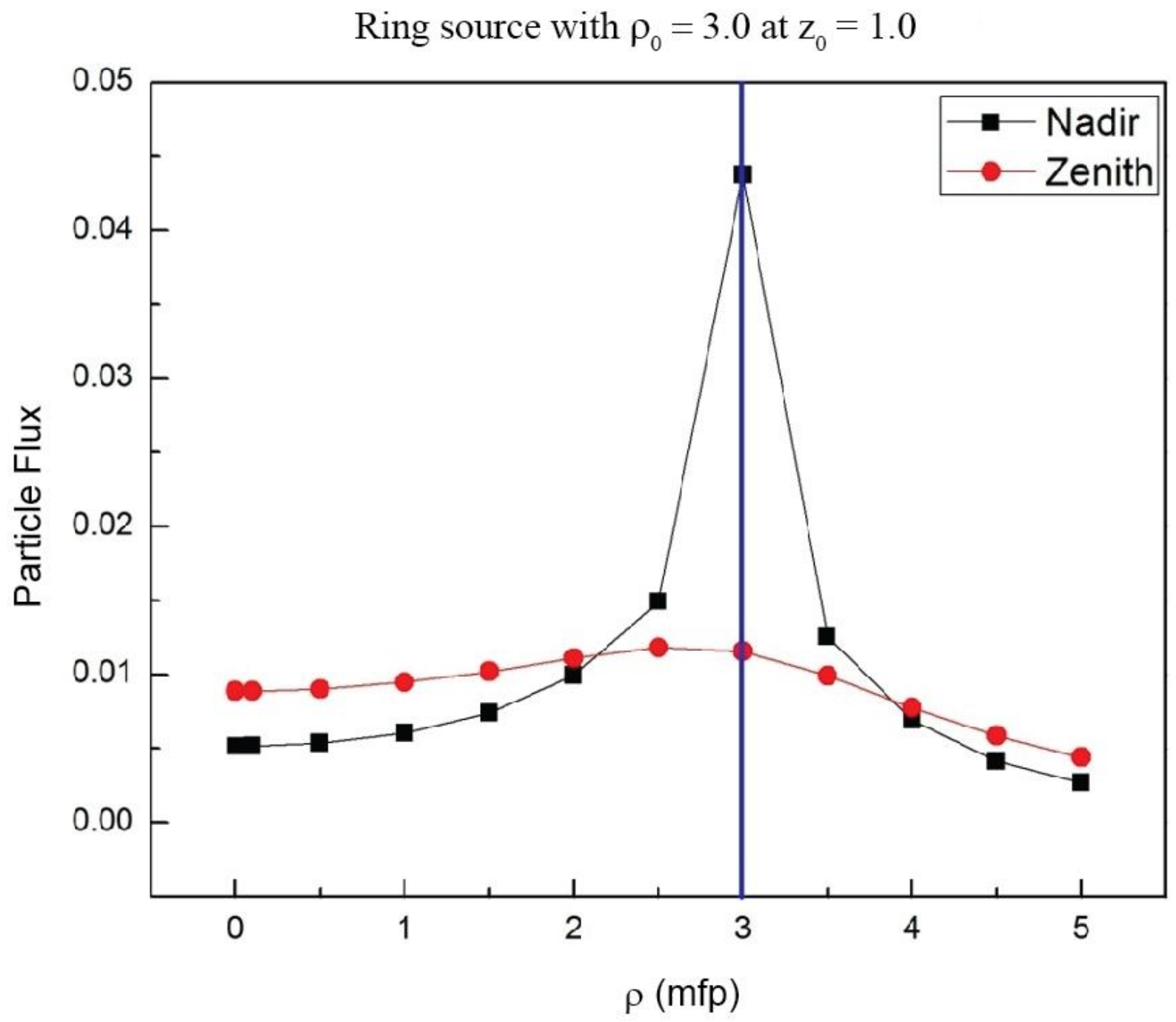

Figure 4: Particle density of ring source for different source directions. 


\subsection{Disk source}

The particle density calculations for a disk source in an infinite bare slab were performed by MCNP and compared with the analytical results for an infinite half space ${ }^{5}$. The infinite bare slab with slab thickness of $b=1000$ mfp was modeled and a mono-directional disk source with radius of $2.0 \mathrm{mfp}$ was put at $\mathrm{z}=1$. The $\mathrm{c}$ value of the medium was set to be 0.99 . The calculation was performed for nadir and zenith cases. The particle density was calculated on the surface $(\mathrm{z}=0)$ with various radius $(\rho=0,0.1,0.5,1.0,1.5,2.0,2.5,3.0,3.5,4.0,4.5$ and 5.0$)$. The f5 detector tally was used for this calculation with detector radius of 0.01 and the number of particles simulated (nps) was $1.0 \times 10^{8}$ for all cases. Table 10 shows the results for both nadir and zenith case. The MCNP calculation results agreed well with the analytic results within 1\%. Figure 5 shows the neutron density for both nadir and zenith cases for the comparison. 
Table 10: Flux for mono-directional disk source (both nadir and zenith) when $\mathbf{c}=\mathbf{0 . 9 9}$.

\begin{tabular}{|c|c|c|c|c|c|c|}
\hline \multirow{2}{*}{$\rho$} & \multicolumn{3}{|c|}{ Nadir } & \multicolumn{3}{|c|}{ Zenith } \\
\hline & $\mathrm{MCNP}(\mathrm{b}=1000)$ & Analytic $(b=\infty)$ & Error $(\%)$ & $\mathrm{MCNP}(\mathrm{b}=1000)$ & Analytic $(b=\infty)$ & Error $(\%)$ \\
\hline 0.0 & 4.8479E-02 & 4.8490E-02 & $-0.02 \%$ & 2.9578E-02 & - & - \\
\hline 0.1 & $4.8496 \mathrm{E}-02$ & 4.8453E-02 & $0.09 \%$ & $2.9531 \mathrm{E}-02$ & $2.9565 \mathrm{E}-02$ & $-0.12 \%$ \\
\hline 0.5 & $4.7531 \mathrm{E}-02$ & $4.7542 \mathrm{E}-02$ & $-0.02 \%$ & $2.8803 \mathrm{E}-02$ & $2.8815 \mathrm{E}-02$ & $-0.04 \%$ \\
\hline 1.0 & 4.4432E-02 & 4.4427E-02 & $0.01 \%$ & $2.6487 \mathrm{E}-02$ & $2.6489 \mathrm{E}-02$ & $-0.01 \%$ \\
\hline 1.5 & 3.8117E-02 & $3.8127 \mathrm{E}-02$ & $-0.03 \%$ & $2.2770 \mathrm{E}-02$ & $2.2767 \mathrm{E}-02$ & $0.01 \%$ \\
\hline 2.0 & $2.4816 \mathrm{E}-02$ & $2.4818 \mathrm{E}-02$ & $-0.01 \%$ & $1.8173 \mathrm{E}-02$ & $1.8173 \mathrm{E}-02$ & $0.00 \%$ \\
\hline 2.5 & $1.2457 \mathrm{E}-02$ & $1.2456 \mathrm{E}-02$ & $0.01 \%$ & $1.3677 \mathrm{E}-02$ & $1.3681 \mathrm{E}-02$ & $-0.03 \%$ \\
\hline 3.0 & $7.2565 \mathrm{E}-03$ & $7.2566 \mathrm{E}-03$ & $0.00 \%$ & $1.0036 \mathrm{E}-02$ & $1.0037 \mathrm{E}-02$ & $-0.01 \%$ \\
\hline 3.5 & $4.5349 \mathrm{E}-03$ & $4.5360 \mathrm{E}-03$ & $-0.02 \%$ & $7.3524 \mathrm{E}-03$ & 7.3497E-03 & $0.04 \%$ \\
\hline 4.0 & $2.9831 \mathrm{E}-03$ & $2.9831 \mathrm{E}-03$ & $0.00 \%$ & $5.4286 \mathrm{E}-03$ & $5.4259 \mathrm{E}-03$ & $0.05 \%$ \\
\hline 4.5 & $2.0402 \mathrm{E}-03$ & $2.0394 \mathrm{E}-03$ & $0.04 \%$ & $4.0530 \mathrm{E}-03$ & $4.0522 \mathrm{E}-03$ & $0.02 \%$ \\
\hline 5.0 & $1.4392 \mathrm{E}-03$ & $1.4379 \mathrm{E}-03$ & $0.09 \%$ & $3.0627 \mathrm{E}-03$ & 3.0633E-03 & $-0.02 \%$ \\
\hline
\end{tabular}




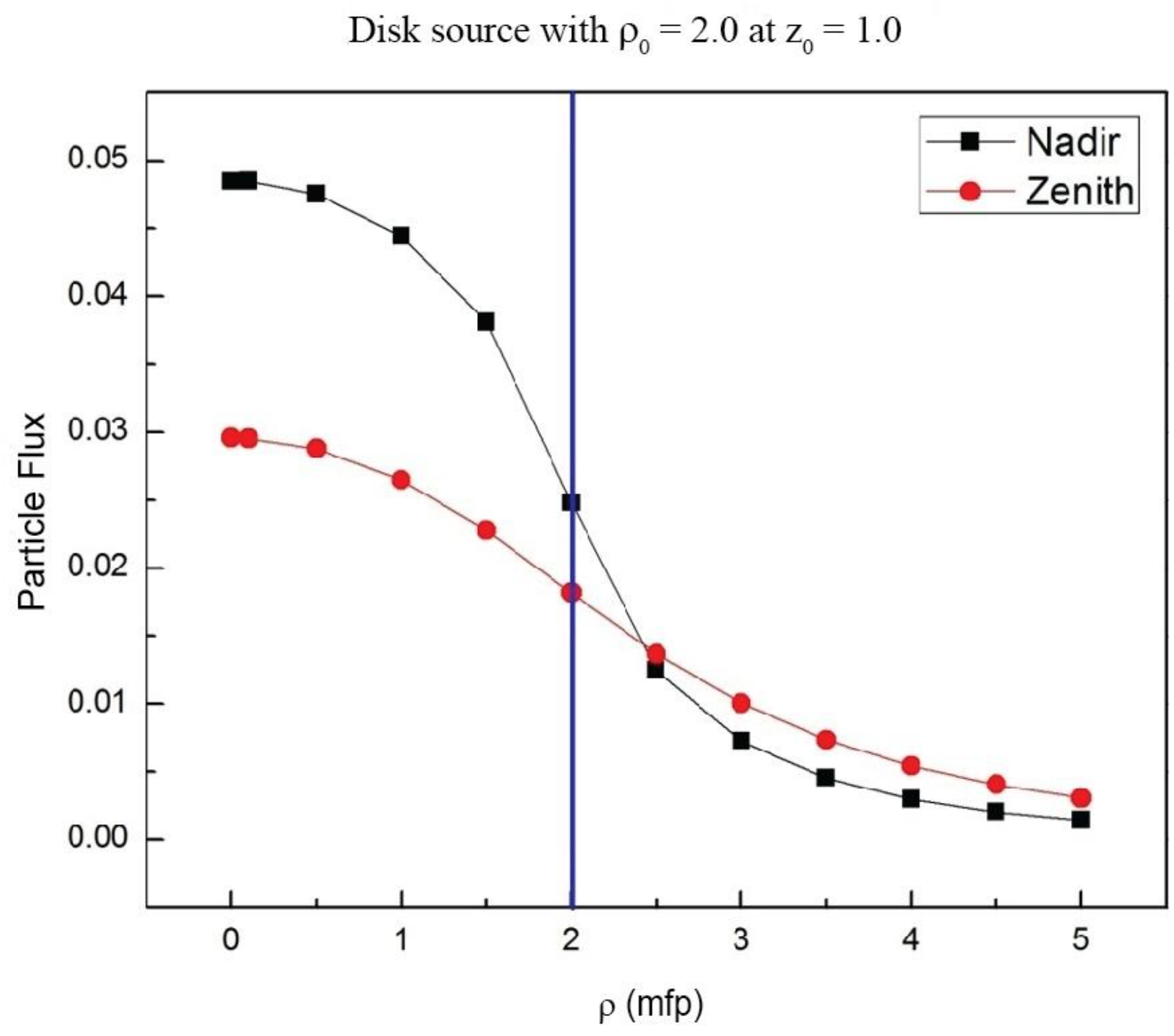

Figure 5. Particle density for different disk source directions. 
We have reported below results for the case $c=1.0$, although analytical results are not available at this point. The calculations were carried out for both nadir and zenith cases with the same conditions except for the number of histories. The number of histories was reduced to $3 \times 10^{7}$ for nadir case and $10^{7}$ for zenith case because of the computing time. 
Table 11. Flux for mono-directional ring source (both nadir and zenith), MCNP, when c=1.0.

\begin{tabular}{c|c|c}
\hline \hline$\rho$ & Nadir (b=1000) & Zenith $(\mathrm{b}=1000)$ \\
\hline 0.0 & $5.0786 \mathrm{E}-02$ & $3.2340 \mathrm{E}-02$ \\
0.1 & $5.0485 \mathrm{E}-02$ & $3.2546 \mathrm{E}-02$ \\
0.5 & $4.9565 \mathrm{E}-02$ & $3.1671 \mathrm{E}-02$ \\
1.0 & $4.6344 \mathrm{E}-02$ & $2.9290 \mathrm{E}-02$ \\
1.5 & $3.9829 \mathrm{E}-02$ & $2.5353 \mathrm{E}-02$ \\
2.0 & $2.6157 \mathrm{E}-02$ & $2.0490 \mathrm{E}-02$ \\
2.5 & $1.3449 \mathrm{E}-02$ & $1.5716 \mathrm{E}-02$ \\
3.0 & $8.0438 \mathrm{E}-03$ & $1.1825 \mathrm{E}-02$ \\
3.5 & $5.1718 \mathrm{E}-03$ & $8.9112 \mathrm{E}-03$ \\
4.0 & $3.5068 \mathrm{E}-03$ & $6.7787 \mathrm{E}-03$ \\
4.5 & $2.4801 \mathrm{E}-03$ & $5.2355 \mathrm{E}-03$ \\
5.0 & $1.8147 \mathrm{E}-03$ & $4.1099 \mathrm{E}-03$ \\
\hline \hline
\end{tabular}




\section{Discussion and Conclusions}

The 2009 paper of Williams is remarkable in both extending the range of analytical solutions made available, and the associated development of techniques, numerical results and analysis near singularities. The final integrals are not easy to evaluate as the integrands are highly oscillatory, singular and also on infinite range. Although the focus of that paper was not on numerical accuracy, the values reported for the problems of point, ring and the disk sources are accurate with rare exceptions (only for the point source, there are some deviations in the last (fifth) digits in five cases out of 196).

We have reported some evaluations of expressions of Williams in this paper, and also compared the results with those of Williams and Ganapol and Kornreich. Overall, the numerical results compare very well, and we are also to explore the nature of the results near singularities in conformity with the results of Williams.

An important purpose of this paper was to verify MCNP-5, the widely used Monte Carlo code against these analytical results. We have found that MCNP is easily able to provide results within about $0.1 \%$ deviation from the "exact" results for most cases, and within $1 \%$ for almost all cases. It is challenged near the singularities, however, where the deviations are much larger, and this should be of some interest as in radiation therapy, for example, the dose should be known well near the sources.

We have not noted CPU times, number of particles, figures of merit etc. for MCNP computations in the tables. We are reporting these for the ring-nadir case in table 12, where we have included results for up to about 10 billion particles. The results converge well, and the final results (the last column) are generally accurate within $0.1 \%$ error, with many of them accurate within $0.05 \%$ error. These results are typical of other cases that we have studied here. 
Table 12: Effect of Increasing the Number of Simulated Particles (NPS) on Accuracy, Simulation Time, and Simulation Figure of Merit.

A number of different simulations were executed for the ring nadir case discussed above, with $\rho_{0}=3.0, z_{0}=1.0, c=0.99$, and $b=128$. The number of simulated particles was then varied over a wide range to examine the effect of increasing particle history counts. It is evident that that very high accuracy is achievable with MCNP. The Figure of Merit (FOM) was computed natively within MCNP by the formula

fom $=2 *(\mathrm{nps} / \mathrm{ctm}$ )(signal-to-noise ratio) . Each tally has its own figure of merit, so for brevity we list the FOM for the tally along $\rho=0.0$. Additionally, the CPU time is reported for the simulations as well.

\begin{tabular}{|c|c|c|c|c|c|c|c|c|c|c|c|}
\hline$\rho$ & & \multicolumn{10}{|c|}{ NPS } \\
\hline$\phi_{\text {analyt. }}$ & & $2^{20}$ & $2^{21}$ & $2^{22}$ & $2^{23}$ & $2^{24}$ & $2^{25}$ & $2^{26}$ & $2^{27}$ & $2^{28}$ & $2^{29}$ \\
\hline 0.0 & $\begin{array}{l}\text { MCNP } \\
\end{array}$ & $\begin{array}{c}5.11852 \mathrm{E}-03 \\
\end{array}$ & $\begin{array}{ll}5.02908 & \text { E-03 }\end{array}$ & $\begin{array}{c}5.10457 \mathrm{E}-03 \\
\end{array}$ & $5.12104 \mathrm{E}-03$ & 5.13145 E-03 & $\begin{array}{l}5.11001 \mathrm{E}-03 \\
\end{array}$ & $25.15448 \mathrm{E}-03$ & $25.15500 \mathrm{E}-03$ & $\begin{array}{c}5.17328 \mathrm{E}-03 \\
\end{array}$ & $\begin{array}{c}5.18062 \mathrm{E}-03 \\
\end{array}$ \\
\hline $5.16470 \mathrm{E}-03$ & Error (\%) & -8.94147 E-01 & $-2.62590 \mathrm{E}+00$ & $-1.16425 \mathrm{E}+00$ & $-8.45354 \mathrm{E}-01$ & -6.43793 E-01 & $-1.05892 \mathrm{E}+00$ & $-1.97882 \mathrm{E}-01$ & $-1.87813 \mathrm{E}-01$ & $1.66128 \mathrm{E}-01$ & $3.08246 \mathrm{E}-01$ \\
\hline 0.1 & MCNP & $5.15052 \mathrm{E}-03$ & $5.08569 \mathrm{E}-03$ & $5.15874 \mathrm{E}-03$ & $5.15468 \mathrm{E}-03$ & $5.17214 \mathrm{E}-03$ & $5.18261 \mathrm{E}-03$ & $5.17235 \mathrm{E}-03$ & $5.17241 \mathrm{E}-03$ & $5.17467 \mathrm{E}-03$ & $5.17726 \mathrm{E}-03$ \\
\hline $5.17285 \mathrm{E}-03$ & Error $(\%)$ & -4.31677 E-01 & $-1.68495 E+00$ & -2.72770 E-01 & $-3.51257 \mathrm{E}-01$ & $-1.37255 \mathrm{E}-02$ & $1.88677 \mathrm{E}-01$ & -9.66585 E- 03 & $-8.50595 \mathrm{E}-03$ & $3.51837 \mathrm{E}-02$ & $8.52528 \mathrm{E}-02$ \\
\hline 0.5 & MCNP & $5.40646 \mathrm{E}-03$ & $5.32357 \mathrm{E}-03$ & $5.33861 \mathrm{E}-03$ & $5.36889 \mathrm{E}-03$ & 5.35859 E-03 & $5.36596 \mathrm{E}-03$ & $5.35816 \mathrm{E}-03$ & $5.36166 \mathrm{E}-03$ & $5.36901 \mathrm{E}-03$ & $5.37161 \mathrm{E}-03$ \\
\hline $5.37202 \mathrm{E}-03$ & Error $(\%)$ & $6.41100 \mathrm{E}-01$ & $-9.01895 \mathrm{E}-01$ & $-6.21926 \mathrm{E}-01$ & -5.82649 E-02 & $-2.49999 \mathrm{E}-01$ & $-1.12807 \mathrm{E}-01$ & $-2.58004 \mathrm{E}-01$ & $-1.92851 \mathrm{E}-01$ & -5.60311 E-02 & $-7.63214 \mathrm{E}-03$ \\
\hline 1.0 & MCNP & $6.14171 \mathrm{E}-03$ & $6.08126 \mathrm{E}-03$ & $6.06195 \mathrm{E}-03$ & $6.05437 \mathrm{E}-03$ & 6.04727 E-03 & $6.04455 \mathrm{E}-03$ & $6.04711 \mathrm{E}-03$ & $6.04838 \mathrm{E}-03$ & $6.04709 \mathrm{E}-03$ & $6.04860 \mathrm{E}-03$ \\
\hline $6.05092 \mathrm{E}-03$ & Error (\%) & $1.50043 \mathrm{E}+00$ & $5.01411 \mathrm{E}-01$ & $1.82286 \mathrm{E}-01$ & $5.70161 \mathrm{E}-02$ & $-6.03214 \mathrm{E}-02$ & $-1.05273 \mathrm{E}-01$ & -6.29656 E-02 & -4.19771 E-02 & -6.32962 E-02 & -3.83413 E-02 \\
\hline 1.5 & MCNP & $7.43894 \mathrm{E}-03$ & 7.41308 E-03 & 7.43049 E-03 & $7.42502 \mathrm{E}-03$ & 7.41915 E-03 & 7.40847 E-03 & $7.40069 \mathrm{E}-03$ & $7.40348 \mathrm{E}-03$ & $7.40345 \mathrm{E}-03$ & $7.40601 \mathrm{E}-03$ \\
\hline $7.40739 \mathrm{E}-03$ & Error $(\%)$ & $4.25926 \mathrm{E}-01$ & $7.68152 \mathrm{E}-02$ & $3.11851 \mathrm{E}-01$ & $2.38006 \mathrm{E}-01$ & 1.58760 E-01 & $1.45800 \mathrm{E}-02$ & -9.04502 E- 02 & $-5.27851 \mathrm{E}-02$ & -5.31901 E-02 & -1.86300 E-02 \\
\hline 2.0 & MCNP & $9.94167 \mathrm{E}-03$ & $9.94051 \mathrm{E}-03$ & $9.94617 \mathrm{E}-03$ & $9.95103 \mathrm{E}-03$ & $9.94530 \mathrm{E}-03$ & $9.94038 \mathrm{E}-03$ & $9.94029 \mathrm{E}-03$ & $9.93549 \mathrm{E}-03$ & $9.93804 \mathrm{E}-03$ & $9.93828 \mathrm{E}-03$ \\
\hline $9.94070 \mathrm{E}-03$ & Error $(\%)$ & $9.75786 \mathrm{E}-03$ & $-1.91133 \mathrm{E}-03$ & $5.50263 \mathrm{E}-02$ & $1.03916 \mathrm{E}-01$ & 4.62744 E-02 & $-3.21909 \mathrm{E}-03$ & $-4.12446 \mathrm{E}-03$ & $-5.24108 \mathrm{E}-02$ & -2.67587 E- 02 & -2.43444 E- 02 \\
\hline 2.5 & MCNP & $1.48919 \mathrm{E}-02$ & $1.49197 \mathrm{E}-02$ & $1.49246 \mathrm{E}-02$ & $1.49357 \mathrm{E}-02$ & $1.49231 \mathrm{E}-02$ & $1.49236 \mathrm{E}-02$ & $1.49262 \mathrm{E}-02$ & $1.49277 \mathrm{E}-02$ & $1.49318 \mathrm{E}-02$ & $1.49307 \mathrm{E}-02$ \\
\hline $1.49319 \mathrm{E}-02$ & Error $(\%)$ & $-2.67883 \mathrm{E}-01$ & $-8.17043 \mathrm{E}-02$ & $-4.88886 \mathrm{E}-02$ & $2.54489 \mathrm{E}-02$ & $-5.89342 \mathrm{E}-02$ & -5.55857 E- 02 & -3.81733 E-02 & -2.81277 E-02 & -6.69707 E-04 & -8.03649 E-03 \\
\hline 3.0 & MCNP & $4.34787 \mathrm{E}-02$ & 4.37270 E-02 & $4.36974 \mathrm{E}-02$ & $4.37680 \mathrm{E}-02$ & $4.37468 \mathrm{E}-02$ & $4.37467 \mathrm{E}-02$ & $4.37323 \mathrm{E}-02$ & $4.37730 \mathrm{E}-02$ & $\infty *$ & $\infty *$ \\
\hline$\infty$ & Error $(\%)$ & Indeterm. & Indeterm. & Indeterm. & Indeterm. & Indeterm. & Indeterm. & Indeterm. & Indeterm. & Exact* & Exact* \\
\hline 3.5 & MCNP & $1.25942 \mathrm{E}-02$ & $1.25532 \mathrm{E}-02$ & $1.25466 \mathrm{E}-02$ & $1.25394 \mathrm{E}-02$ & $1.25314 \mathrm{E}-02$ & $1.25412 \mathrm{E}-02$ & $1.25428 \mathrm{E}-02$ & $1.25411 \mathrm{E}-02$ & $1.25390 \mathrm{E}-02$ & $1.25375 \mathrm{E}-02$ \\
\hline $1.25368 \mathrm{E}-02$ & Error $(\%)$ & $4.57852 \mathrm{E}-01$ & $1.30815 \mathrm{E}-01$ & 7.81699 E-02 & $2.07389 \mathrm{E}-02$ & -4.30732 E-02 & 3.50967 E-02 & 4.78591 E-02 & $3.42990 \mathrm{E}-02$ & $1.75483 \mathrm{E}-02$ & $5.58356 \mathrm{E}-03$ \\
\hline 4.0 & MCNP & $6.88993 \mathrm{E}-03$ & $6.88750 \mathrm{E}-03$ & $6.89036 \mathrm{E}-03$ & $6.88680 \mathrm{E}-03$ & $6.88874 \mathrm{E}-03$ & $6.89672 \mathrm{E}-03$ & $6.90080 \mathrm{E}-03$ & $6.90305 \mathrm{E}-03$ & $6.90622 \mathrm{E}-03$ & $6.90775 \mathrm{E}-03$ \\
\hline $6.90711 \mathrm{E}-03$ & Error (\%) & -2.48729 E-01 & -2.83910 E-01 & $-2.42504 \mathrm{E}-01$ & $-2.94045 \mathrm{E}-01$ & -2.65958 E-01 & $-1.50425 \mathrm{E}-01$ & -9.13551 E- 02 & $-5.87800 \mathrm{E}-02$ & -1.28853 E-02 & $9.26581 \mathrm{E}-03$ \\
\hline 4.5 & MCNP & $4.16294 \mathrm{E}-03$ & $4.15359 \mathrm{E}-03$ & $4.15741 \mathrm{E}-03$ & $4.15688 \mathrm{E}-03$ & 4.15754 E-03 & $4.16122 \mathrm{E}-03$ & $4.16353 \mathrm{E}-03$ & $4.16400 \mathrm{E}-03$ & $4.16490 \mathrm{E}-03$ & $4.16546 \mathrm{E}-03$ \\
\hline $4.16513 \mathrm{E}-03$ & Error $(\%)$ & -5.25794 E-02 & -2.77062 E-01 & $-1.85348 \mathrm{E}-01$ & -1.98073 E-01 & -1.82227 E-01 & -9.38746 E- 02 & $-3.84142 \mathrm{E}-02$ & $-2.71300 \mathrm{E}-02$ & -5.52204 E- 03 & $7.92292 \mathrm{E}-03$ \\
\hline 5.0 & MCNP & $2.67266 \mathrm{E}-03$ & $2.66176 \mathrm{E}-03$ & $2.66513 \mathrm{E}-03$ & $2.66866 \mathrm{E}-03$ & $2.66822 \mathrm{E}-03$ & $2.67284 \mathrm{E}-03$ & $2.67491 \mathrm{E}-03$ & $2.67397 \mathrm{E}-03$ & $2.67359 \mathrm{E}-03$ & $2.67234 \mathrm{E}-03$ \\
\hline $2.67187 \mathrm{E}-03$ & Error $(\%)$ & $2.95673 \mathrm{E}-02$ & -3.78387 E-01 & $-2.52258 \mathrm{E}-01$ & $-1.20141 \mathrm{E}-01$ & -1.36608 E-01 & $3.63042 \mathrm{E}-02$ & $1.13778 \mathrm{E}-01$ & $7.85966 \mathrm{E}-02$ & $6.43744 \mathrm{E}-02$ & $1.75907 \mathrm{E}-02$ \\
\hline & CPU time (min) & 50.9 & 54.32 & 57.71 & 64.49 & 78.31 & 105.85 & 276.77 & 4746.41 & 8663.64 & 16768.33 \\
\hline & fom & $6.162 \mathrm{E}+01$ & $1.459 \mathrm{E}+02$ & $2.360 \mathrm{E}+02$ & $1.549 \mathrm{E}+02$ & $2.922 \mathrm{E}+02$ & $4.036 \mathrm{E}+02$ & $2.985 \mathrm{E}+02$ & $3.822 \mathrm{E}+00$ & $6.487 \mathrm{E}+00$ & $4.080 \mathrm{E}+00$ \\
\hline
\end{tabular}

* Note that MCNP effectively treats values $>10^{34}$ as infinite

The computing environment used was a Sony VAIO® laptop with the Windows-7 64 bit OS, with an Intel ${ }^{\circledR}$ Core ${ }^{\mathrm{TM}}$ i7-3612QM CPU with a clock speed of $2.10 \mathrm{GHz}$ and $8 \mathrm{~Gb}$ RAM. MCNP was operated in threading mode, the times indicated are with respect to MCNP threading over 8 cores; physical computing time was much shorter. 


\section{Acknowledgements}

Sudarshan Loyalka would like to thank Professor Williams for giving him the opportunity to work on the problem, many helpful discussions and encouragement. He also thanks Professor Ganapol for sharing his results and computer programs. Jeho Jeong and Nathan White were partially supported, respectively, by the University of Missouri Nuclear Science and Engineering Institute and a GAANN grant from the U.S. Department of Education. This work has also been partially supported by a NEUP-2982 grant from the U.S. Department of Energy. 


\section{References}

1. M. M. R. Williams, "Three-dimensional transport theory: an analytical solution of an internal beam searchlight problem, I”, Annals of Nuclear Energy, 36(8), 1256-1261 (2009).

2. Barry D. Ganapol and Drew E. Kornreich, “Three-dimensional transport theory: An analytical solution for the internal beam searchlight problem, II", Annals of Nuclear Energy, 36(8), 1242-1255 (2009).

3. Forrest Brown and Nathan Barnett, "A Tutorial on Using MCNP for 1-Group Transport Calculations", Los Alamos National Laboratory report, LA-UR-07-4594 (2006).

4. S. Naz and S. K. Loyalka, "One-speed criticality problems for a bare slab and sphere: Some benchmark results", Annals of Nuclear Energy 34(9), 693-702 (2007).

5. S. K. Loyalka and M. M. R. Williams, "Computation of some transport theory expressions for a line source in an infinite half-space", Annals of Nuclear Energy, 36(5), 612-619 (2009).

6. Levin, D. "Fast integration of rapidly oscillatory functions. " Journal of Computational and Applied Mathematics 67 (1996): 95-101 